\title{
La familia Cactaceae en Argentina: patrones de diversidad y PRIORIDADES POLÍTICAS PARA SU CONSERVACIÓN
}

\author{
PABLO ORTEGA-BAES ${ }^{1}$, HÉCTOR GODÍNEZ-ALVAREZ², JESÚS SAJAMA ${ }^{1}$, PABLO \\ GOROSTIAGUE ${ }^{1}$, SILVIA SÜHRING ${ }^{1}$, GUADALUPE GALÍNDEZ1 ${ }^{1}$, SILVIA BRAVO ${ }^{1}$, DIEGO \\ LÓPEZ-SPAHR ${ }^{1}$, MARIANA ALONSO-PEDANO ${ }^{1}$, LUCÍA LINDOW-LÓPEZ ${ }^{1}$, ANDREA \\ BARRIONUEVO ${ }^{1}$, CECILIA SOSA ${ }^{1}$, RAMIRO N. CURTI' ${ }^{1}$ y ALEJANDRA JUÁREZ1
}

\begin{abstract}
Resumen: En este trabajo se utilizó la riqueza de especies y el endemismo de las especies de cactáceas argentinas para seleccionar las provincias con la mayor prioridad para la conservación de esta familia de plantas en el país. Además, se evaluó el papel de la diversidad de cactáceas como sustitutos de la diversidad de plantas dicotiledóneas de Argentina. La riqueza de especies (número de especies) y el endemismo (número de especies endémicas) fueron determinadas para cada una de las 24 provincias de Argentina. Un total de 15 provincias fueron seleccionadas como prioritarias con base en todas las especies de cactáceas y 14 con base en las especies endémicas. La provincia de Salta presenta la mayor riqueza de especies y endemismo. Las especies endémicas son un buen sustituto de la diversidad de cactáceas. De igual manera, las cactáceas son un buen sustituto de la diversidad de las plantas dicotiledóneas.
\end{abstract}

Palabras clave: Endemismo, riqueza de especies, Salta, sustituto.

\begin{abstract}
Summary: The Cactaceae family in Argentina: diversity patterns and political priorities for their conservation. In this paper we used species richness and endemism of Argentine cacti to select those provinces with highest priority for the conservation of this plant family in the country. The role of cactus diversity as a surrogate for dicot diversity was further analyzed. Species richness (number of species) and endemism (number of endemic species) were determined for each of the 24 provinces of Argentina. Fifteen provinces were selected as priority based on the number of species and 14 provinces were selected based on the number of endemic species. Salta province presents the highest species richness and endemism. Endemic species are a good surrogate for cactus diversity. Similarly, cactus diversity is a good surrogate for dicot diversity.
\end{abstract}

Key words: Endemism, Salta province, species richness, surrogate.

\section{INTRODUCCIÓN}

La selección de áreas prioritarias para acciones de conservación tiene como propósito principal, enfocar las acciones de conservación en un número limitado de áreas que tienen un valor especial por el tipo de biodiversidad que ellas contienen (Mittermeier et al., 1997; Myers, 2001). Entre las

1 Laboratorio de Investigaciones Botánicas (LABIBO), Facultad de Ciencias Naturales, Universidad Nacional de Salta- CONICET, Av. Bolivia 5150, Salta 4400, Argentina, E-mail: ortega_baes@yahoo.com.ar

2 UBIPRO, FES-Iztacala, UNAM, Av. de los Barrios 1, Los Reyes Iztacala, Tlalnepantla 54090, Edo. de México, Ap. postal 314, México aproximaciones utilizadas a escala global o regional podemos destacar la que considera como unidad de análisis a países o territorios políticos (Ceballos \& Brown, 1995; Caldecott et al., 1996; Mittemeier et al., 1997; Lira et al., 2002; Dávila-Aranda et al., 2004; Ortega-Baes \& Godínez-Alvarez, 2006; Godínez-Alvarez \& Ortega-Baes, 2007). Esta aproximación se basa en el hecho de que aunque la distribución de la biodiversidad no reconoce límites políticos, las acciones atinentes a su conservación se realizan en el marco de la soberanía que los territorios políticos tienen sobre sus recursos biológicos y que les permite soportar su desarrollo (Caldecott et al., 1996; Mittermeier et al., 1997; Villaseñor et al., 1998; Sarukhán \& Dirzo, 2001; Ortega-Baes \& Godínez-Alvarez, 2006). 
Las cactáceas son elementos característicos del continente Americano que se distribuyen principalmente en las regiones áridas y semiáridas. México es el país con la mayor diversidad, seguido por Brasil, Argentina y Bolivia, en cuyos territorios se encuentran los principales centros de diversidad reconocidos para la familia (Ortega-Baes \& Godínez-Alvarez, 2006; Ortega-Baes et al., 2010). Actualmente, muchas especies de cactáceas están amenazadas a escala global debido al avance de la frontera agropecuaria, la urbanización, el desarrollo de infraestructura, la minería, la colecta y el comercio ilegal y potencialmente por el cambio climático (Ortega-Baes et al., 2010). Debido a su valor ornamental, el comercio internacional ha tenido un efecto negativo sobre la conservación de las cactáceas por lo que muchas especies están incluidas en el Apéndice I de CITES y toda la familia está incluida en el Apéndice II (Oldfield, 1997; Ortega-Baes et al., 2010). De igual manera, muchas especies de cactáceas han sido recientemente incorporadas a las listas internacionales de especies en riesgo de extinción (IUCN 2013).

Argentina es uno de los países prioritarios para la conservación de la familia Cactaceae debido a su alta riqueza específica y a sus altos niveles de endemismo (Ortega-Baes \& Godínez-Alvarez, 2006, OrtegaBaes et al. 2010). Las cactáceas están ampliamente distribuidas en este país, en donde habitan regiones biogeográficas como la Prepuna, la Puna, el Monte, el Chaco y la Patagonia (Mourelle \& Ezcurra, 1996; Ortega-Baes et al., 2012). La región del noroeste del país es la más diversa y forma parte de uno de los centros de diversidad de cactáceas más importantes a escala global (Ortega-Baes et al., 2010), siendo los valles calchaquíes una de las áreas geográficas más diversa de esta región (Ortega-Baes et al., 2012). Otra área de alta diversidad se encuentra en el Este del país, que junto con Paraguay, Uruguay y el sur de Brasil constituyen un centro de diversidad para esta familia (Oldfield, 1997; Ortega-Baes et al., 2010). Aun cuando Argentina es un país prioritario para la conservación de este grupo de plantas, no se han definido dentro del mismo, áreas o regiones de importancia para este propósito.

Las provincias en Argentina tienen soberanía sobre sus recursos naturales (artículo 41 y 124; Constitución Nacional, 1994), por lo que una estrategia nacional para la conservación de la diversidad en general y de las cactáceas en particular debería tener como primer paso definir las provincias con la mayor importancia relativa para este propósito. La definición de estas provincias sin embargo depende de la realización de inventarios de diversos grupos de organismos para así poder identificar aquellas provincias que presentan la mayor diversidad biológica. La realización de los mismos es una tarea difícil debido a la gran cantidad de tiempo y recursos que son necesarios. En este sentido, la utilización de grupos sustitutos de la diversidad puede ser una alternativa útil para la identificación de áreas prioritarias. Un grupo de organismos puede ser considerado como un buen sustituto cuando las áreas seleccionadas con base en su diversidad capturan una alta proporción de la diversidad regional objeto de conservación (Howard et al., 1998; Reyers et al., 2000; Williams et al., 2006).

El propósito del presente trabajo fue analizar la diversidad de la familia Cactaceae en Argentina para determinar las provincias que tienen prioridad para la conservación de este grupo de especies. Además, se evaluó el papel de las especies endémicas como sustitutos de toda la diversidad de cactáceas. Recientemente, se ha demostrado que a escala regional las especies endémicas son un buen sustituto de la diversidad total de especies de esta familia de plantas (Ortega-Baes et al., 2012). Esto se debe a la alta proporción de endemismo político que ha sido registrada en esta familia (Ortega-Baes \& Godínez-Álvarez, 2006; GodínezÁlvarez \& Ortega-Baes, 2007). Por lo tanto, se esperaba que también a escalas mayores (país), las especies endémicas tuvieran un buen desempeño como sustituto de la diversidad de cactáceas. Por último, dada la importancia de las cactáceas como grupo focal para acciones de conservación, se analizó su papel como sustituto de la diversidad total de las plantas dicotiledóneas de Argentina. Si las cactáceas tuvieran un buen desempeño como sustituto, acciones de conservación definidas para este grupo redundarían a favor de la conservación de una alta proporción de la diversidad de plantas dicotiledóneas de este país.

\section{Materiales y Métodos}

\section{Base de datos}

La base de datos fue elaborada con base en 
Kiesling et al. (2008). Esta propuesta taxonómica difiere de otros autores (Hunt, 2006), pero se decidió utilizarla debido a que es la única fuente que incluye información sobre la diversidad taxonómica y la distribución geográfica de todas las cactáceas en las 24 provincias del país. Esta base de datos forma parte de una compilación sobre la diversidad de las plantas vasculares del Cono Sur (Zuloaga et al., 2008). Con esta información se elaboraron dos matrices, una para todas las especies de cactáceas (excepto las especies exóticas) y otra para las especies endémicas, en las que se registró la presenciaausencia de cada especie en cada provincia. Se consideraron como especies endémicas aquellas que sólo se distribuyen en Argentina (endemismo político; Cowling, 2001). Con estas matrices se determinó el número de especies (riqueza específica) y el número de especies endémicas (endemismo) por provincia.

\section{Provincias prioritarias para la conservación}

La selección de las provincias prioritarias para la conservación de las cactáceas se realizó utilizando el programa ResNet (Reserve Network; Garson et al., 2007). ResNet es una aplicación que implementa variaciones de algoritmos propuestos por Margules et al. (1988), basándose en tres principios básicos: rareza, complementariedad y riqueza. Con base en la rareza, los sustitutos de la biodiversidad son ordenados inversamente de acuerdo a la frecuencia de su ocurrencia en el grupo de datos. Con posterioridad, los sitios (en nuestro caso provincias) de la región a planificar son ordenados de acuerdo a si ellos registran el sustituto más raro, el siguiente más raro y así sucesivamente. Para que se cumpla el supuesto de complementariedad, los sitios son ordenados con base en el número de sustitutos que no han cumplido con el nivel de representación del objetivo inicial. Por último, la riqueza es considerada para ordenar los sitios de acuerdo al número de especies presentes (Garson et al. 2007). Los análisis fueron hechos utilizando todas las especies (TE) y las especies endémicas (EE) por separado, seleccionando provincias hasta que todas las especies de cactáceas estuvieron representadas al menos una vez. El programa Surrogacy (Garson \& Sarkar, 2002) fue usado para evaluar el papel de las especies endémicas de cactáceas como sustitutos de toda la diversidad de cactáceas de Argentina. Este programa utiliza el programa ResNet para seleccionar las áreas del sustituto (EE) y determina la proporción acumulada de la diversidad que se desea representar. Además, calcula la representatividad de la diversidad en áreas seleccionadas al azar, asumiendo que todas las áreas (provincias en nuestro caso) tienen la misma probabilidad de ser seleccionadas en cada etapa, determinando los límites de confianza para esa representatividad. El desempeño del sustituto fue evaluado teniendo en cuenta la representatividad de la diversidad objeto de conservación (todas las especies de cactáceas de Argentina) y su efectividad frente a áreas seleccionadas al azar (1000 simulaciones).

Las cactáceas como sustituto de la diversidad de plantas dicotiledóneas de Argentina

Se utilizaron todas las especies (TE) y las especies endémicas (EE) de cactáceas para evaluar su utilidad como sustitutos de toda la diversidad de plantas dicotiledóneas y de las especies endémicas de este grupo de plantas. Todos los análisis se realizaron utilizando el programa Surrogacy (Garson \& Sarkar, 2002). La información sobre la presencia o ausencia de cada especie de planta dicotiledónea (nativas y endémicas) en cada provincia argentina fue obtenida del Catálogo de las Plantas Vasculares del Cono Sur (Zuloaga et al., 2008).

\section{Resultados}

\section{Riqueza específica y endemismo}

La familia Cactaceae en Argentina estuvo representada por 238 especies, de las cuales 133 especies fueron endémicas. Las cactáceas se distribuyeron en 22 provincias de Argentina (Fig. 1A). La provincia de Salta registró el número más alto de especies (86), seguida por Jujuy (68) y Catamarca (57). Salta también presentó el mayor número de especies endémicas (44), seguida por La Rioja (35) y Catamarca (35; Fig. 1B). Las provincias de Chubut y Santa Cruz presentaron el número más bajo de especies (Fig. 1A), mientras que Chaco y Misiones no presentaron especies endémicas (Fig. 1B). 

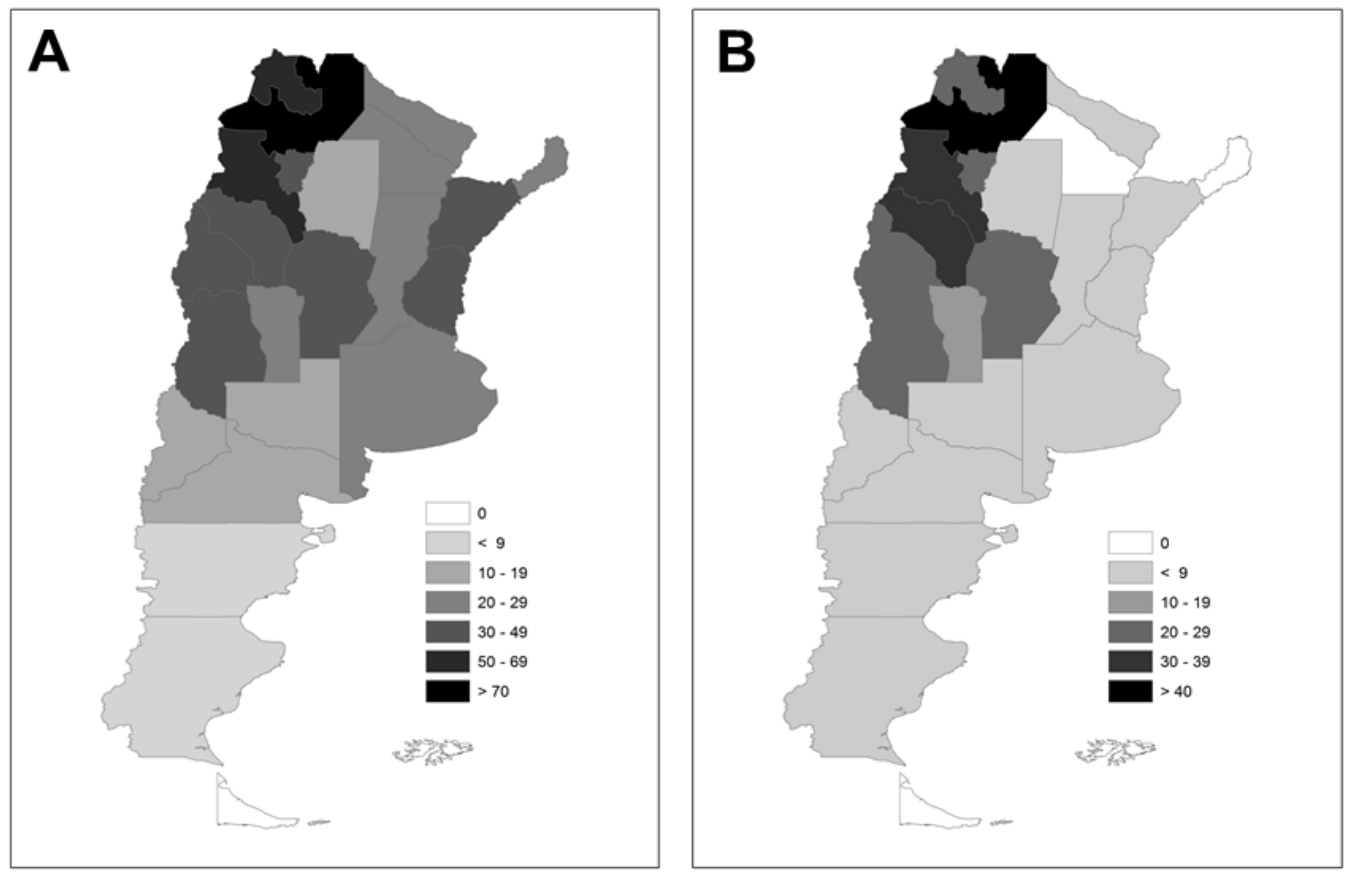

Fig. 1. Distribución geográfica de la riqueza específica (A) y el endemismo (B) de la familia Cactaceae por provincias en Argentina.

Provincias prioritarias para la conservación

Quince provincias de acuerdo con el análisis TE y 14 de acuerdo con el análisis EE fueron seleccionadas como prioritarias para la conservación de las cactáceas en Argentina (Fig. 2). La congruencia entre ambos análisis fue alta, con el $93 \%$ de las provincias compartidas (Fig. 2). Las provincias de Salta y La Rioja fueron las más importantes tanto en el análisis TE como en el análisis EE, debido a que albergan el $48 \%$ del total de las especies y el $49 \%$ de las especies endémicas de cactáceas del país (Fig. 2).

Cuando se evaluó el papel de las especies endémicas como sustitutos de la diversidad de cactáceas, las provincias seleccionadas presentaron un mejor desempeño que las provincias seleccionadas al azar (Fig. 2). La representatividad alcanzó el $64 \%$ con las cinco primeras provincias seleccionadas y el $98 \%$ con el total de provincias elegidas (Fig. 2).

Las cactáceas como sustituto de la diversidad de plantas dicotiledóneas de Argentina

Cuando se utilizaron las cactáceas (análisis TE y EE) como sustitutos de la diversidad de las

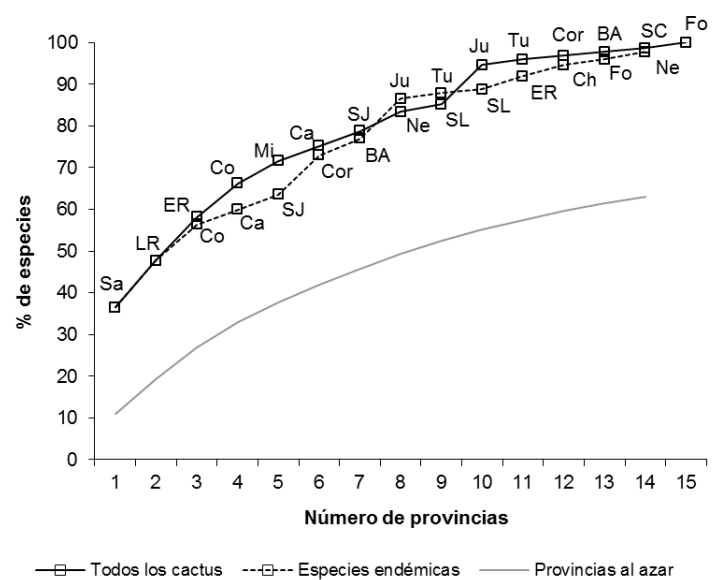

Fig. 2. Porcentaje acumulado de especies representadas en cada una de las 15 provincias seleccionadas como prioritarias usando todas las especies y desempeño de las especies endémicas como sustitutos de toda la diversidad de cactáceas. La línea gris muestra el desempeño de las provincias seleccionadas al azar. BA: Buenos Aires, Ca: Catamarca, Cha: Chaco, Co: Corrientes, Cor: Córdoba, ER: Entre Ríos, Fo: Formosa, Ju: Jujuy, LR: La Rioja, Mi: Misiones, Ne: Neuquén, Sa: Salta, SC: Santa Cruz, SJ: San Juan, SL: San Luis y Tu: Tucumán. 
plantas dicotiledóneas (todas las especies y las especies endémicas) las provincias seleccionadas se desempeñaron mejor que provincias seleccionadas al azar (Fig. 3 y 4). Sin embargo, el mejor desempeño se registró para el análisis TE. Para todas las especies de dicotiledóneas, la representatividad de las 14 provincias seleccionadas por el análisis TE fue del $95 \%$, mientras que con el mismo número de provincias seleccionadas por el análisis EE fue del $87 \%$. Cuando se evaluó el desempeño de las cactáceas como sustituto de las especies endémicas de dicotiledóneas, la representatividad fue del $92 \%$ para el análisis TE y del $90 \%$ para el análisis EE.

\section{Discusıón}

De acuerdo con Ortega-Baes \& Godínez-Alvarez (2006) y Ortega-Baes et al. (2010), Argentina es un país prioritario para la conservación de cactáceas debido a su alta riqueza de especies y endemismo. Sin embargo, hasta ahora no se había realizado

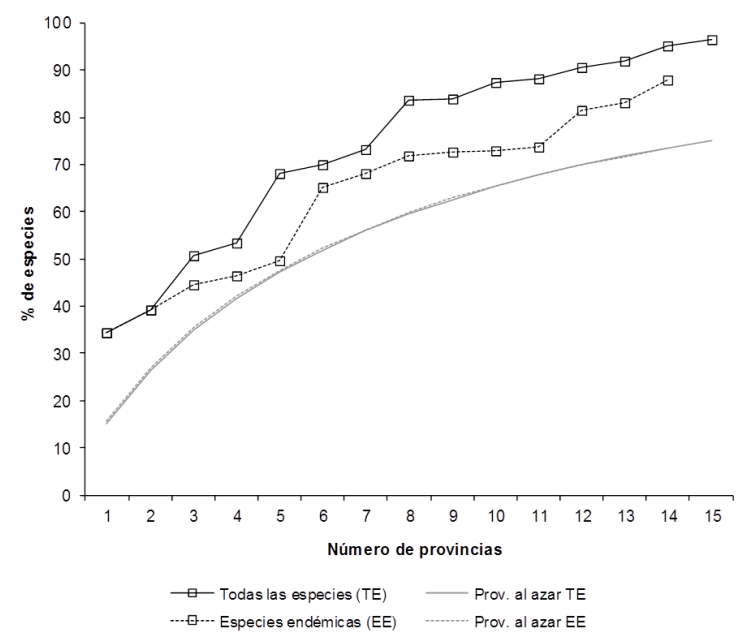

Fig. 3. Porcentaje acumulado de especies de plantas dicotiledóneas representadas en las provincias seleccionadas con base en todas las especies de cactáceas (TE) y en las especies endémicas de cactáceas (EE). La línea gris continua muestra el desempeño de las provincias seleccionadas al azar de acuerdo a todas las especies de cactáceas y la línea gris punteada muestra el desempeño de las provincias seleccionadas al azar de acuerdo a las especies endémicas de cactáceas. ninguna evaluación para determinar las prioridades de conservación en este país, siendo esta la primera propuesta para este grupo de plantas en Argentina.

Las cinco provincias con el más alto número de especies (en orden de importancia Salta, Jujuy, Catamarca, La Rioja y Tucumán) pertenecen a la región noroeste. Esta región es considerada parte del segundo centro de diversidad más importante a escala global para la familia Cactaceae (Oldfield, 1997; Ortega-Baes et al., 2012) y una de las dos regiones con la más alta riqueza de especies de plantas de Argentina (Zuloaga et al., 1999; Juárez et al., 2007). De acuerdo con los resultados obtenidos, esta región es también el centro más importante de endemismo en el país para este grupo de plantas, por lo tanto debería ser considerado como una región prioritaria para la conservación de cactáceas en Argentina.

Los resultados indican que Salta es la provincia con la más alta riqueza de especies y endemismo de cactáceas en el país. En esta provincia se registró el $33 \%$ de las cactáceas endémicas argentinas,

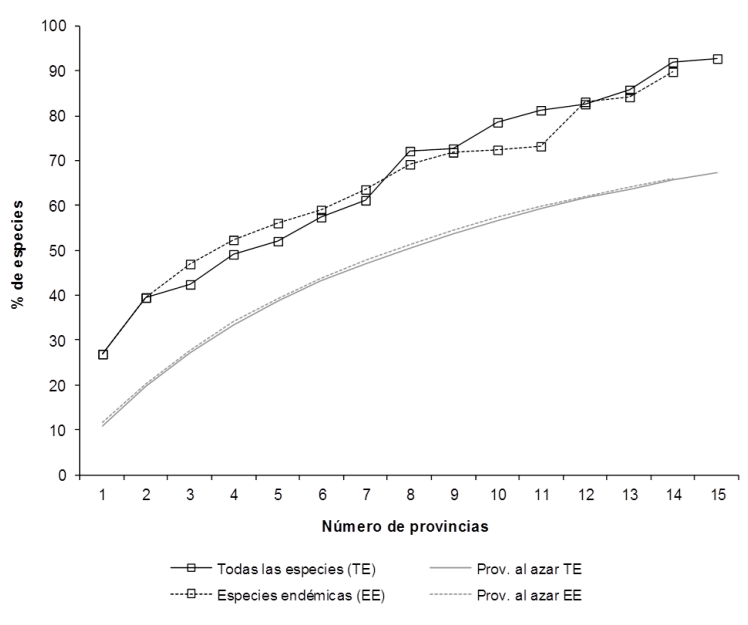

Fig. 4. Porcentaje acumulado de las especies endémicas de plantas dicotiledóneas representadas en las provincias seleccionadas con base en todas las especies de cactáceas (TE) y en las especies endémicas de cactáceas (EE). La línea gris continua muestra el desempeño de las provincias seleccionadas al azar de acuerdo a todas las especies de cactáceas y la línea gris punteada muestra el desempeño de las provincias seleccionadas al azar de acuerdo a las especies endémicas de cactáceas. 
con 12 especies endémicas exclusivas a esta provincia. Algunas de las especies de cactáceas que se distribuyen en Salta enfrentan altos niveles de amenaza debido al cambio en el uso de la tierra (por ejemplo, Echinopsis albispinosa y Stetsonia coryne; Ruiz, 2011), a la colecta ilegal (por ejemplo, E. ancistrophora y Blossfeldia liliputana; Cavalli-Cabrera, 2009) y a la extracción de madera (por ejemplo, E. atacamensis y E. terscheckii; Ortega, 2010). Esta provincia debe ser considerada como "megadiversa" y con alta prioridad para la conservación de la biodiversidad en Argentina, no sólo por las cactáceas sino también por la diversidad de otros grupos de organismos (Mares et al., 1989; Zuloaga et al., 1999; Juárez et al., 2007).

Los resultados mostraron que existe una alta congruencia espacial entre las provincias seleccionadas con base en todas las especies de cactáceas y las seleccionadas por las especies endémicas. Esto puede explicarse por la relación positiva existente entre la riqueza de especies y el endemismo en la familia Cactaceae (Ortega-Baes \& Godínez-Álvarez, 2006; Godínez-Álvarez \& Ortega-Baes, 2007; Ortega-Baes et al., 2010). Como fue indicado a escala regional (Ortega-baes et al., 2012), existió una alta representatividad cuando se utilizaron las cactáceas endémicas como grupo indicador de toda la diversidad de cactáceas de Argentina.

La selección de provincias prioritarias con base en ResNet, indican que Salta es la provincia más importante a la que se deben sumar las provincias de La Rioja y Catamarca en la región noroeste y las provincias de Entre Ríos, Corrientes y Misiones en la región noreste del país. Estas provincias forman parte de los dos centros más importantes reconocidos a escala global, que incluyen a la diversidad de Argentina (Oldfield, 1997; Ortega-Baes et al., 2010). Además, los resultados muestran que provincias ubicadas en otras regiones geográficas del país son importantes para la conservación de la diversidad de cactáceas de Argentina. Este es el caso de Córdoba y San Juan, las que fueron seleccionadas con alta prioridad con base en el endemismo.

En el presente estudio se registró una alta representatividad de las especies de plantas dicotiledóneas en las áreas seleccionadas con base en la diversidad de cactáceas. Teniendo en cuenta la escala de análisis utilizada (grano grueso), los resultados indican que una estrategia basada en un grupo prioritario como las cactáceas, puede ser efectiva además para toda la diversidad de plantas dicotiledóneas de Argentina. Futuros estudios deberían evaluar a escala fina la importancia de las cactáceas como sustitutos de la diversidad en Argentina. Además, deberían analizarse si las provincias seleccionadas como prioritarias en el presente trabajo también lo son para otros grupos de organismos.

Los resultados del presente trabajo muestran que no todas las provincias tienen la misma importancia relativa para la conservación de la biodiversidad de cactáceas en nuestro país. Algunas son más importantes que otras con base en la singularidad de su riqueza de especies y en sus niveles de endemismo político. Esto pone de relieve la necesidad de realizar esfuerzos de conservación en las provincias seleccionadas como prioritarias, sobre todo en aquellas que están enfrentando altos niveles de amenazas. Este es el caso de la provincia de Salta, la que se ha visto fuertemente afectada por el avance de la frontera agropecuaria debido al proceso de sojización (Volante et al., 2005). Las acciones de conservación implementadas para este grupo de plantas en Argentina (al igual que en otros países de Latinoamérica) son aún escasas (OrtegaBaes et al. 2010). Por lo tanto, se necesita establecer de manera inmediata estrategias in situ y ex situ que permitan conservar, a largo plazo, la singular flora de cactáceas de este país.

\section{Agradecimientos}

El estudio fue subsidiado por el CIUNSalta (PI 1957 y 2060), CONICET (PIP 01149) y FONCYT (PICT 1492).

\section{Bibliografía}

CALDECOTT, J. O., M. D. JENKINS, T. H. JOHNSON \& B. GROOMBRIDGE. 1996. Priorities for conserving global species richness and endemism. Biodivers. Conserv. 5: 699-727.

CAVALLI-CABRERA, A. 2009. Diagnóstico sobre extracción y venta de cactus en la provincia de Salta (Argentina): especies importantes y actores 
involucrados. Tesis de Ingeniería en Recursos Naturales y Medio Ambiente, Facultad de Ciencias Naturales, Universidad Nacional de Salta, Salta.

CEBAllos, G. \& J. H. BROWN. 1995. Global patterns of mammalian diversity, endemism, and endangerment. Conserv. Biol. 9: 559-568.

COWLlinG, R. M. 2001. Endemism. In: Levin, S. A. (ed.), Encyclopedia of Biodiversity, pp. 497-507. San Diego Academics, San Diego.

DÁVILA-ARANDA, P., R. LIRA-SAADE \& J. VALDÉS-REYNA. 2004. Endemic species of grasses in Mexico: a phytogeographic approach. Biodivers. Conserv. 13: 1101-1121.

GARSON, J. \& S. SARKAR. 2002. Surrogacy Manual. Version 1.1. Available from http://uts.cc.utexas. edu/ consbio/Cons/program.html.

GARSON, J., A. AGGARWAL \& S. SARKAR. 2007. ResNet (Version 1.2) Manual. Biodiversity and Biocultural Conservation Laboratory, Section of Integrative Biology, University of Texas, Austin.

GODÍNEZ-ALVAREZ, H. \& P. ORTEGA-BAES. 2007. Mexican cactus diversity: environmental correlates and conservation priorities. Bol. Soc. Bot. Mex. 81: 81-87.

HOWARD, P.C., P. VISKANIC, T. R. B. DAVENPORT, F. W. KIGENYI, M. BALTZER, C. DICKINSON, J. S. LWANGA, R. A. MATTHEWS \& A. BALMFORD. 1998. Complementarity and the use of indicator groups for reserve selection in Uganda. Nature 394: 472-475.

HUNT, D. 2006. The new cactus lexicon. Remous Ltd. Milborne Port, Somerset.

IUCN. 2013. The IUCN Red List of Threatened Species. Version 2014.3. <www.iucnredlist.org>. Downloaded on 29 January 2015.

JUÁREZ, A., P. ORTEGA-BAES, S. SÜHRING, W. MARTÍN \& G. GALÍNDEZ. 2007. Spatial patterns of dicot diversity in Argentina. Biodivers. Conserv. 16: 1669-1677.

KIESLING, R., J. LAROCCA, L. FAÚNDEZ, D. METZING \& S. ALBESIANO. 2008. Cactaceae. In: Zuloaga, F. O, O. Morrone \& M. J. Belgrano (eds.), Catálogo de las Plantas Vasculares del Cono Sur. Monogr. Missouri Bot. Garden. 107: 1715-1830.

LIRA, R., J. L. VILLASEÑOR \& E.ORTIZ. 2002. A proposal for the conservation of the family Cucurbitaceae in México. Biodivers. Conserv. 11: 1699-1720.

MARES, M. A., R. A. OJEDA \& R. M. BARQUEZ. 1989. Guide to the mammals of Salta province, Argentina. University of Oklahoma Press, Oklahoma.

MARGULES, C. R., A. O. NICHOLLS \& R. L. PRESSEY. 1988. Selecting networks of reserves to maximize biological diversity. Biol. Conserv. 43: $63-76$.
MitTERMEIER, R. A., P. R. GIL \& C. G. MITTERMEIER. 1997. Megadiversidad. CEMEX, México.

MOURELLE, C. \& E. EZCURRA. 1996. Species richness of Argentine cacti: a test of biogeographic hypotheses. J. Veget. Sci. 7: 667-680.

MYERS, N. 2001. Hotsposts. In: Levin, S. A. (ed.). Encyclopedia of Biodiversity: 371-381. San Diego Academics, San Diego.

OLDFIELD, S. (Compiler). 1997. Cactus and succulent plants: status survey and conservation action plan. IUCN/SSC cactus and succulent specialist group. International Union for Conservation of Nature and Natural resources, Gland and Cambridge.

ORTEGA, A. 2010. Evaluación poblacional de dos especies de cactus utilizadas como fuente de madera en la Provincia de Salta (Argentina). Tesis de Ingeniería en Recursos Naturales y Medio Ambiente, Facultad de Ciencias Naturales, Universidad Nacional de Salta, Salta.

ORTEGA-BAES, P. \& H. GODÍNEZ-ALVAREZ. 2006. Global diversity and conservation priorities in the Cactaceae. Biodiver. Conserv. 15: 817-827.

ORTEGA-BAES, P., S. SÜHRING, J. SAJAMA, E. SOTOLA, M. ALONSO-PEDANO, S. BRAVO \& H. GODÍNEZ-ALVAREZ. 2010. Diversity and conservation in the cactus family. In: RAMAWAT, K. G. (ed.) Desert Plants: 157-173. Springer, BerlinHeidelberg.

ORTEGA-BAES, P., S. BRAVO, J. SAJAMA, S. SÜHRING, J. ARRUETA, E. SOTOLA, M. ALONSO-PEDANO, A.C. GODOY-BÜRKI, N.R. FRIZZA, G. GALÍNDEZ, P. GOROSTIAGUE, A. BARRIONUEVO \& A. SCOPEL. 2012. Intensive field surveys in conservation planning: Priorities for cactus diversity in the Saltenian Calchaquíes Valleys (Argentina). J. Arid Environ. 82: 91-97.

REYERS, B., A. S. VAN JAARSVELD \& M. KRÜGER. 2000. Complementarity as a biodiversity indicator strategy. Proc. Roy. Soc. London B Biol. 267: 505513.

RUIZ, S. 2011. ¿Están las cactáceas del Noroeste de Argentina amenazadas por el cambio en el uso del suelo? Tesis de Ingeniero en Recursos Naturales y Medio Ambiente, Facultad de Ciencias Naturales, Universidad Nacional de Salta, Salta.

SARUKHÁN, J. \& R. DIRZO. 2001. Biodiversity-rich countries. In: Levin, S. A. (ed.). Encyclopedia of Biodiversity: 419-436. San Diego Academics Press, San Diego.

VILLASEÑOR, J. L., G. IBARRA \& D. OCAÑA. 1998. Strategies for the conservation of Asteraceae in México. Conserv. Biol. 12: 1066-1075.

VOLANTE, J. N., A.R. BIANCHI, H.P. PAOLI, Y.E. NOÉ, H.J. ELENA \& C.M. CABRAL. 2005. 
Bol. Soc. Argent. Bot. 50 (1) 2015

Análisis de la dinámica del uso del suelo agrícola del Noroeste argentino mediante teledetección y sistemas de información geográfica. Período 20002005. INTA EEA, Salta.

WILLIAMS, P., D. FAITH, L. MANNE, W. SECHREST \& C. PRESTON. 2006. Complementarity analysis: Mapping the performance of surrogates for biodiversity. Biol. Conserv. 128: 253-264.

ZULOAGA, F. O., O. MORRONE \& D. RODRÍGUEZ. 1999. Análisis de la Biodiversidad en plantas vasculares de la Argentina. Kurtziana 27: 17-167.
ZULOAGA, F. O., O. MORRONE, \& M. J. BELGRANO. 2008. Catálogo de las Plantas Vasculares del Cono Sur. Monogr. Syst. Bot. Missouri Bot. Garden 107.

Recibido el 4 de agosto de 2014, aceptado el 4 de febrero de 2015 . 